\title{
Second harmonic generation for collagen I characterization in rectal cancer patients with and without preoperative radiotherapy
}

Stéphanie Blockhuys

Nisha Rani Agarwal

Camilla Hildesjö

Ingvar Jarlsfelt

Pernilla Wittung-Stafshede

Xiao-Feng Sun 


\title{
Second harmonic generation for collagen I characterization in rectal cancer patients with and without preoperative radiotherapy
}

\author{
Stéphanie Blockhuys, ${ }^{a, b, c, *}$ Nisha Rani Agarwal, ${ }^{c, \dagger}$ Camilla Hildesjö, ${ }^{a, b, d}$ Ingvar Jarlsfelt, ${ }^{e}$ \\ Pernilla Wittung-Stafshede, ${ }^{c}$ and Xiao-Feng Sun ${ }^{a, b, *}$ \\ aLinköping University, Department of Oncology, Linköping, Sweden \\ bLinköping University, Department of Clinical and Experimental Medicine, Linköping, Sweden \\ 'Chalmers University of Technology, Department of Biology and Biological Engineering, Gothenburg, Sweden \\ dUniversity Hospital of Linköping, Department of Clinical Pathology, Linköping, Sweden \\ ${ }^{e}$ Ryhov Hospital, Department of Pathology, Jönköping, Sweden
}

\begin{abstract}
Rectal cancer is treated with preoperative radiotherapy $(R T)$ to downstage the tumor, reduce local recurrence, and improve patient survival. Still, the treatment outcome varies significantly and new biomarkers are desired. Collagen I (Col-I) is a potential biomarker, which can be visualized label-free by second harmonic generation (SHG). Here, we used SHG to identify Col-I changes induced by RT in surgical tissue, with the aim to evaluate the clinical significance of RT-induced Col-I changes. First, we established a procedure for quantitative evaluation of Col-I by SHG in CDX2-stained tissue sections. Next, we evaluated Col-I properties in material from 31 non-RT and 29 RT rectal cancer patients. We discovered that the Col-I intensity and anisotropy were higher in the tumor invasive margin than in the inner tumor and normal mucosa, and RT increased and decreased the intensity in inner tumor and normal mucosa, respectively. Furthermore, higher Col-I intensity in the inner tumor was related to increased distant recurrence in the non-RT group but to longer survival in the RT group. In conclusion, we present a new application of SHG for quantitative analysis of Col-I in surgical material, and the first data suggest Col-I intensity as a putative prognostic biomarker in rectal cancer. @ The Authors. Published by SPIE under a Creative Commons Attribution 3.0 Unported License. Distribution or reproduction of this work in whole or in part requires full attribution of the original publication, including its DOI. [DOI: 10.1117/1.JBO.22.10.106006]
\end{abstract}

Keywords: collagen I; second harmonic generation; prognosis; rectal cancer; radiotherapy.

Paper 170387R received Jun. 13, 2017; accepted for publication Sep. 12, 2017; published online Oct. 10, 2017.

\section{Introduction}

Colorectal cancer (CRC) is the third most common cancer worldwide with around 1.36 million new incidences reported in 2012. About 700,000 estimated deaths from CRC make it the fourth most common cause of cancer-related deaths worldwide accounting for about $8.5 \%$. The incidence rate for rectal cancer is about $30 \%$ from all CRC cases, and today's 5-year survival rate for rectal cancer is around $63 \% .^{1,2}$ Surgery is the mainstay of curative treatments for rectal cancer patients. ${ }^{3}$ Preoperative radiotherapy (RT) is now commonly used to downstage the tumor, reduce the risk of local recurrence, and improve the overall survival in rectal cancer patients. ${ }^{4,5}$ Although the 5-year survival rate for rectal cancer has increased around $20 \%$ during the last three decades due to earlier diagnosis and improved treatments, less than half of the patients achieve a complete pathological response after preoperative chemoradiotherapy. ${ }^{6}$ Therefore, it is essential to find new molecular markers to give a better prognosis after treatment and to achieve better treatment outcome.

The tumor is spatially heterogeneous, ${ }^{7,8}$ whereby the tumor extracellular matrix (ECM) has an emerging role in cancer

\footnotetext{
*Address all correspondence to: Stéphanie Blockhuys, E-mail: steblo@ chalmers.se; Xiao-Feng Sun, E-mail: xiao-feng.sun@liu.se

${ }^{\dagger}$ Current address: McMaster University, Biointerfaces Institute and Department
} of Chemistry and Chemical Biology, Hamilton, Ontario, Canada progression and treatment response. ${ }^{9,10}$ Hereby, the most abundant ECM protein, collagen I (Col-I), is recognized as a dynamic and active substrate that may not only block but also stimulate cancer cell invasion and metastasis, which is the main reason for cancer deaths. ${ }^{11}$ During cancer progression, Col-I progressively thickens, stiffens, and linearizes. Hereby, linearized crosslinked collagen bundles stimulate cell migration, whereas a dense network of cross-linked matrix fibers blocks migration. ${ }^{12}$ Furthermore, the signature of thicker and radial aligned Col-I fibers was assigned as a prognostic factor for reduced survival in breast cancer. ${ }^{13}$ Fibrosis is a side-effect of RT, which involves increased collagen deposition and remodeling, mediated by direct and indirect interactions between the tumor's cellular component and the collagen matrix. ${ }^{14,15}$ RT may stimulate cancer metastasis via multiple mechanisms, which imply responses by both the tumor cells and tumor stroma. ${ }^{16,17}$ Earlier, we reported that RT stimulates colon cancer cells to remodel the Col-I matrix into straps, i.e., aligned Col-I fibers, associated with an increased cell migration potential. ${ }^{18}$ Still, detailed information on the RT-induced changes in the Col-I matrix and their clinical significance remain unclear.

Hence, quantitative and morphological characterization of the Col-I matrix at the microscale becomes important in the evaluation of prognosis and treatment outcome. This information can be obtained by second harmonic generation (SHG): a nonlinear microscopy (NLM) technique. ${ }^{19}$ NLM can provide label-free information by two- or multiphoton processes in the 
interaction of short-pulsed laser with molecules and their vibrations. Low absorption and scattering of near-infrared (NIR) excitation light by the tissue allows deep imaging into tissue sections ( 0.4 to $1 \mathrm{~mm}$ depending on tissue structure). SHG arises from the laser field suffering a conserved nonlinear, secondorder, polarization when passing through noncentrosymmetric structures. Col-I is such a noncentrosymmetric protein detected by SHG using an excitation wavelength of 800 to $860 \mathrm{~nm}$ and an emission wavelength that is half the excitation wavelength. ${ }^{20}$

Although Col-I properties have been associated with CRC progression, ${ }^{21-23}$ the effects of RT on the Col-I matrix in rectal cancers and their clinical relevance remain unknown. In this study, we characterized the Col-I matrix by SHG in CDX2stained surgical tissue sections from 60 patients that participated in a Swedish rectal cancer clinical trial of preoperative RT, and we examined the relationship of the collected Col-I properties in different subtumor regions with the clinical outcome. This information is particularly interesting considering the application of SHG-coupled colonoscopy in the evaluation of treatment outcome of rectal cancer patients.

\section{Materials and Methods}

\subsection{Patient Material}

The patients were from the South-East Swedish Health Care region and participated in the randomized Swedish clinical trial of preoperative RT during 1987 to $1990 .{ }^{24}$ Each participant signed the informed consent. The patient cohort included 60 primary rectal adenocarcinomas, among them, 31 patients underwent surgery only and 29 patients underwent RT before surgery. RT was administered with 25 Gy in five fractions during a median of 7 days (range, 4 to 8 days). Surgery was then performed within a median of 4 days (range, 1 to 8 days) after RT. None of the patients received adjuvant chemotherapy before or after surgery. The mean age of the patients was 68 years (range, 39 to 79 years), and the median follow-up was 79 months (range, 0 to 301 months). Other patient and tumor characteristics are presented in Table 1.

\subsection{CDX2 Immunohistochemical Staining}

Four micrometer thin sections from formalin-fixed, paraffinembedded (FFPE) surgical tissue were baked in $65^{\circ} \mathrm{C}$ for $2 \mathrm{~h}$, followed by deparaffinization in Aqua de Par 10× Ancillary reagent $\left(\mathrm{ADP} 1002 \mathrm{M}\right.$, Biocare $\mathrm{Medical}^{1}$ ) for $20 \mathrm{~min}$. The sections were then submitted to Borg Decloaker RTU antigen retrieval solution ${ }^{1}$ (BD1000) for heat-induced epitope retrieval using a pressure cooker Decloaking Chamber NxGen ${ }^{1}$ programmed in a temperature cycle to reach a maximum of $110^{\circ}$ $\mathrm{C}$ for $5 \mathrm{~min}$. After cooling down, the slides were rinsed in tap water prior to washing in tris-buffered saline (TBS) wash buffer. After blocking endogenous peroxidase by incubation in peroxidazed $1^{1}$ for $5 \mathrm{~min}$, the slides were washed in TBS wash buffer and incubated with the monoclonal primary antibody to CDX2 [Clone DAK-CDX2 (Agilent, GA080)] for $30 \mathrm{~min}$ at room temperature (RT) followed by incubation with secondary antibody MACH4 Universal horseradish peroxidase (HRP)-Probe and tertiary antibody MACH4 Universal HRP-Polymer ${ }^{1}$ (BRR 4012) for $10 \mathrm{~min}$ each at RT. After HRP detection, the tissue section was rinsed in TBS wash buffer. For visualization, one drop $(32 \mu \mathrm{l})$ of diaminobenzidine (DAB) chromogen per $1.0 \mathrm{ml}$ of DAB substrate buffer ${ }^{1}$ was applied to
Table 1 Patient and tumor characteristics.

\begin{tabular}{|c|c|c|}
\hline Characteristics & Non-RT (\%) & $\mathrm{RT}(\%)$ \\
\hline \multicolumn{3}{|l|}{ Gender } \\
\hline Male & $20(65)$ & $19(66)$ \\
\hline Female & $11(35)$ & $10(34)$ \\
\hline \multicolumn{3}{|l|}{ Age (years) } \\
\hline$\leq 69$ & $12(39)$ & $14(48)$ \\
\hline$>69$ & $19(61)$ & $15(52)$ \\
\hline \multicolumn{3}{|l|}{ Tumor stage } \\
\hline I & $11(35)$ & $9(31)$ \\
\hline II & $8(26)$ & $9(31)$ \\
\hline III & $12(39)$ & $11(38)$ \\
\hline IV & $0(0)$ & $0(0)$ \\
\hline \multicolumn{3}{|l|}{ Differentiation } \\
\hline Well & $3(10)$ & $2(7)$ \\
\hline Moderate & $22(71)$ & $17(59)$ \\
\hline Poor & $6(19)$ & $8(27)$ \\
\hline Unknown & 0 & $2(7)$ \\
\hline \multicolumn{3}{|l|}{ Surgical type } \\
\hline Rectal amputation & $17(55)$ & $10(34)$ \\
\hline Abdominoperineal resection & $15(45)$ & $19(66)$ \\
\hline \multicolumn{3}{|l|}{ Resection margin } \\
\hline Tumor free & $28(90)$ & $28(96)$ \\
\hline Tumor positive & $3(10)$ & $1(4)$ \\
\hline \multicolumn{3}{|l|}{ To anal verge $(\mathrm{cm})$} \\
\hline Mean & 7.2 & 8.8 \\
\hline
\end{tabular}

the tissue sections followed by incubation for $5 \mathrm{~min}$ at RT. After rinsing in deionized water, the sections were counterstained with Mayers hematoxylin staining solution (Histolab Products $\mathrm{AB}^{2}$ ) and mounted with Pertex mounting media. ${ }^{2}$

\subsection{Second Harmonic Generation Microscopy}

The 532-nm (frequency doubled) beam of the Nd: $\mathrm{YVO}_{4}$ pump laser (picoTRAIN, HighQ GmbH) is used to pump the optical parametric oscillator (OPO), (Levante Emerald OPO, APE $\mathrm{GmbH}$ ) to provide an output of $817 \mathrm{~nm}$. The pump beam has a pulse length of $7 \mathrm{ps}$ and repetition rate of $76 \mathrm{MHz}$. The $817-\mathrm{nm}$ beam is coupled into the scanning head unit (Nikon C1) of the Nikon Eclipse TE2000-E microscope. The beam was focused on the sample using a high NA oil objective (Nikon Plan Fluor $40 \times / 1.30$ ). SHG signal was collected in transmission with a high NA lens into a single-photon counting photomultiplier tube (HPM-100-40, Becker \& Hickl GmbH). SHG signals were 
collected at $408.5 \mathrm{~nm}$ with bandpass filters 405/10. Single acquisitions were taken in $83 \mathrm{~s}$ with a size of 1024 pixels for a $318.3 \mu \mathrm{m}$ field-of-view.

SHG signals were calibrated every time before a measurement. The calibration was carried out using a sugar crystal preserved for all measurements. The counts were recorded for $1 \mathrm{~s}$ collection time on the same region chosen on the crystal ranged from 25 to 30 .

\subsection{Collagen-I Characterization}

Col-I was characterized for the global properties Col-I intensity and anisotropy and for the local individualistic properties Col-I fiber number, length, and width. Col-I intensity and anisotropy were analyzed using ImageJ software. Col-I intensity was calculated by dividing the sum of all intensities by the total number of pixels. The Col-I anisotropy was measured using the ImageJ plugin, FibrilTool. ${ }^{25}$ Col-I fiber number, length, and width were analyzed using CT-FIRE software. ${ }^{26}$ CT-FIRE analysis was applied to raw SHG images with parameter values threshim $2=0$, number of selected scales $=4$, and the rest of the parameters having default values. The Col-I fiber number data were corrected for the percentages of surface covered by ECM, quantified by applying ImageJ software to the phase-contrast images of the regions of interest (RoIs), i.e., the tissue regions selected for SHG imaging.

\subsection{Statistics}

The student's $t$-test was applied to determine the statistical significance of the difference in Col-I properties results in surgical samples from non-RT versus RT treated rectal cancer patients. All Col-I properties results are presented as mean \pm standard error. For clinical analysis, the raw Col-I data were categorized into low ( $\leq$ median) and high $(>$ median $)$ values. The Chi-square test was applied to examine the relationships of the Col-I properties with clinicopathological variables. Logrank test was used to examine the relationships of the Col-I properties with the relative risk for (local or distant) recurrence and the patient (overall or disease-free) survival. Stratified log rank test was used for examining independency of the Col-I properties from treatment (non-RT versus RT) in the disease recurrence and patient survival. Survival curves were computed according to the Kaplan-Meier method. The tests were twosided and $P$-values of $<0.05$ were considered as significant (SPSS Statistics version 22.0).

\section{Results and Discussion}

\subsection{Experimental Setup for Col-I Characterization by SHG in Surgical Tissue}

We established a new experimental setup for the evaluation of Col-I in surgical tissue from Swedish rectal cancer clinical trial of preoperative RT during 1987 to 1990 . Col-I was visualized label-free by SHG microscopy in CDX2-stained tissue sections. SHG images were acquired in different regions of the tissue, namely adjacent normal mucosa, inner tumor, and invasive margin. For each tissue region, three separate SHG images were taken [Fig. 1(a)]. A phase-contrast scan of the CDX2-stained tissue section was used to preselect the RoIs and to relocate the RoIs by bright field view before SHG image acquisition [Fig. 1(b)].

With this setup, label-free and quantitative evaluation of the Col-I matrix in FFPE surgical tissue material was obtained. SHG in comparison to more classic Col-I staining approaches, e.g., (a)

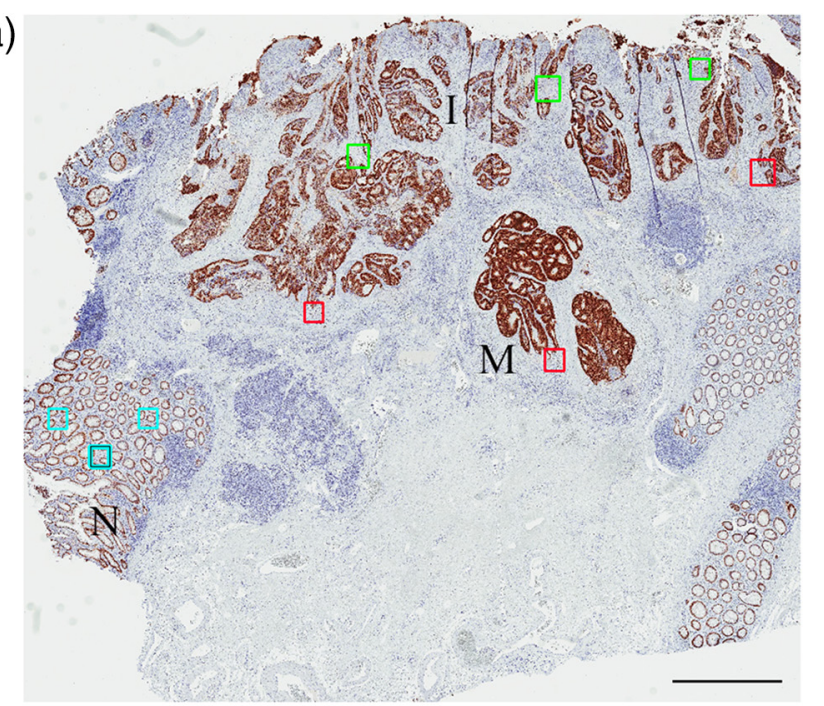

(b)

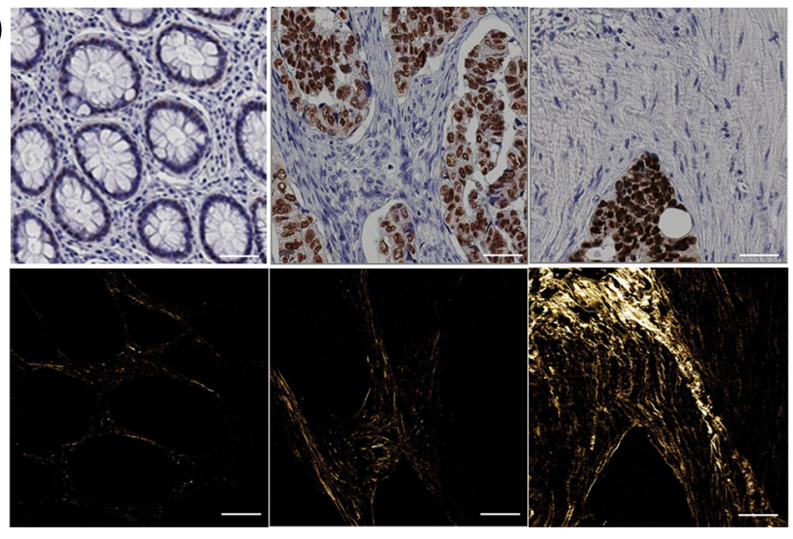

Fig. 1 Visualization of Col-I in CDX2-stained rectal surgical sections by SHG. (a) Phase-contrast scan of a CDX2-stained tissue section with indication of the regions of interest in adjacent normal mucosa ( $\mathrm{N}$; blue squares), inner tumor (l; green squares), and tumor invasive margin ( $\mathrm{M}$; red squares). Scale bar $=1 \mathrm{~mm}$. (b) Phase-contrast (upper panels) and SHG (lower panels) images from a Rol in normal mucosa (left panels), inner tumor (middle panels), and invasive margin (right panels) regions of a CDX2-stained rectal surgical section. Scale bars $=50 \mu \mathrm{m}$.

Masson's trichrome or sirius red staining, provides information about not only the amount but also the architecture of the Col-I matrix. ${ }^{19,27}$ Others have analyzed the Col-I matrix using SHG in normal mucosa and tumor tissue, ${ }^{21,22}$ but we, for the first time, evaluated the Col-I matrix in adjacent normal mucosa, inner tumor, and invasive margin regions in the samples, thereby analyzing region-specific aspects of tumor development. The CDX2 staining is important as it allows for specific visualization of the rectal epithelial cells, ${ }^{28}$ whereas the hematoxylin and eosin staining, used by others to correlate the SHG signal to the tumor epithelium, visualizes the nuclei of all cell types. ${ }^{13,22}$ Our setup may be used for the investigation of the direct interplay between the Col-I matrix and epithelial cells upon combining SHG with two-photon emission fluorescence microscopy, which allows for colocalization of Col-I and epithelial cells in the tissue.

\subsection{Col-I Characterization in Surgical Tissue from non-RT and RT Treated Patients}

We determined Col-I intensity and Col-I anisotropy in the normal mucosa, inner tumor, and invasive margin of CDX2-stained 
surgical tissue sections from 31 non-RT and 29 RT rectal cancer patients [Fig. 2(a)]. While inner tumor and invasive margin were present in all 60 tissues (31 non-RT and 29 RT), the adjacent normal mucosa was only found in 24 of the 60 tissues (12 non-RT and 12 RT). For the non-RT group, we observed no difference between normal mucosa and inner tumor, but higher intensity and anisotropy in the invasive margin versus normal mucosa and inner tumor $(p<0.001)$. Analysis of the effect of RT on the Col-I properties in the different tissue regions showed that RT is related to a decreased $(p=0.006)$ and increased $(p=0.044)$ intensity in the normal mucosa and inner tumor, respectively. Furthermore, RT did not affect the intensity in the invasive margin or the anisotropy in any of the tissue regions. We also quantified other Col-I fiber properties, such as number, length, and width in the different tissue regions under non-RT and RT conditions [Fig. 2(b)]. Comparison of the different tissue regions showed higher fiber number, length, and width in invasive margin versus normal mucosa and inner tumor ( $p<0.001$ for all). RT was related to a decreased fiber number $(p=0.005)$ in the normal mucosa and decreased fiber width in the invasive margin $(p=0.022)$, respectively.

Several studies have reported a pivotal role of collagen in colon cancer progression. ${ }^{21-23}$ Birk et al. ${ }^{21}$ described an increased amount and alignment of Col-I in malignant versus normal colonic mucosa. However, we found no difference for these two Col-I properties between inner tumor and normal mucosa. Kalluri and Zeisberg ${ }^{29}$ report that the invasive margin has a different cellular microenvironment with more fibroblasts that produce and reorganize the Col-I matrix, which may be a possible explanation for our observation of an increased Col-I amount and alignment in the invasive margin. Because our study is the first report on preoperative RT-induced Col-I changes in patient tumor material, detailed comparisons to others' data are not possible. Other findings have indicated differences in matrix metalloproteinases production with matrix degradation ${ }^{30}$ and fibroblast activity with matrix production, ${ }^{14}$ which may explain the decreased Col-I amount in the normal mucosa and increased Col-I amount in the inner tumor, respectively. The discovered differences in RT effect between Col-I amount and fiber number for inner tumor and normal mucosa regions suggest an increased amount per Col-I fiber, indicating a higher ordered structure of the Col-I fibrils. ${ }^{19,31}$ The latter observation exemplifies the strength of using SHG for a detailed study of Col-I microscopic properties in tissue material.

\subsection{Col-I Characteristics in Relation to Clinicopathological Variables}

We evaluated the clinical relevance of the Col-I properties intensity and anisotropy by determining their relationships with different clinicopathological variables, including tumor stage, the grade of differentiation, recurrence, and survival. In the non-RT group, we found that the Col-I intensity in the inner tumor was positively related to both the grade of differentiation $(p=0.018)$ and distant recurrence [ $p=0.003$; Fig. 3(a)]. Furthermore, we discovered a trend toward higher overall survival for non-RT patients with higher intensity in the invasive margin [ $p=0.064$; Fig. 3(b)]. In the RT group, we observed a trend of higher overall survival for RT patients with higher intensity in the inner tumor $[p=0.077$; Fig. 3(c)]. Besides, there were no significant correlations between the Col-I properties and other clinicopathological variables. Further evaluation of the correlation between the Col-I fiber length and width, and the stage and differentiation grade of the disease indicated a
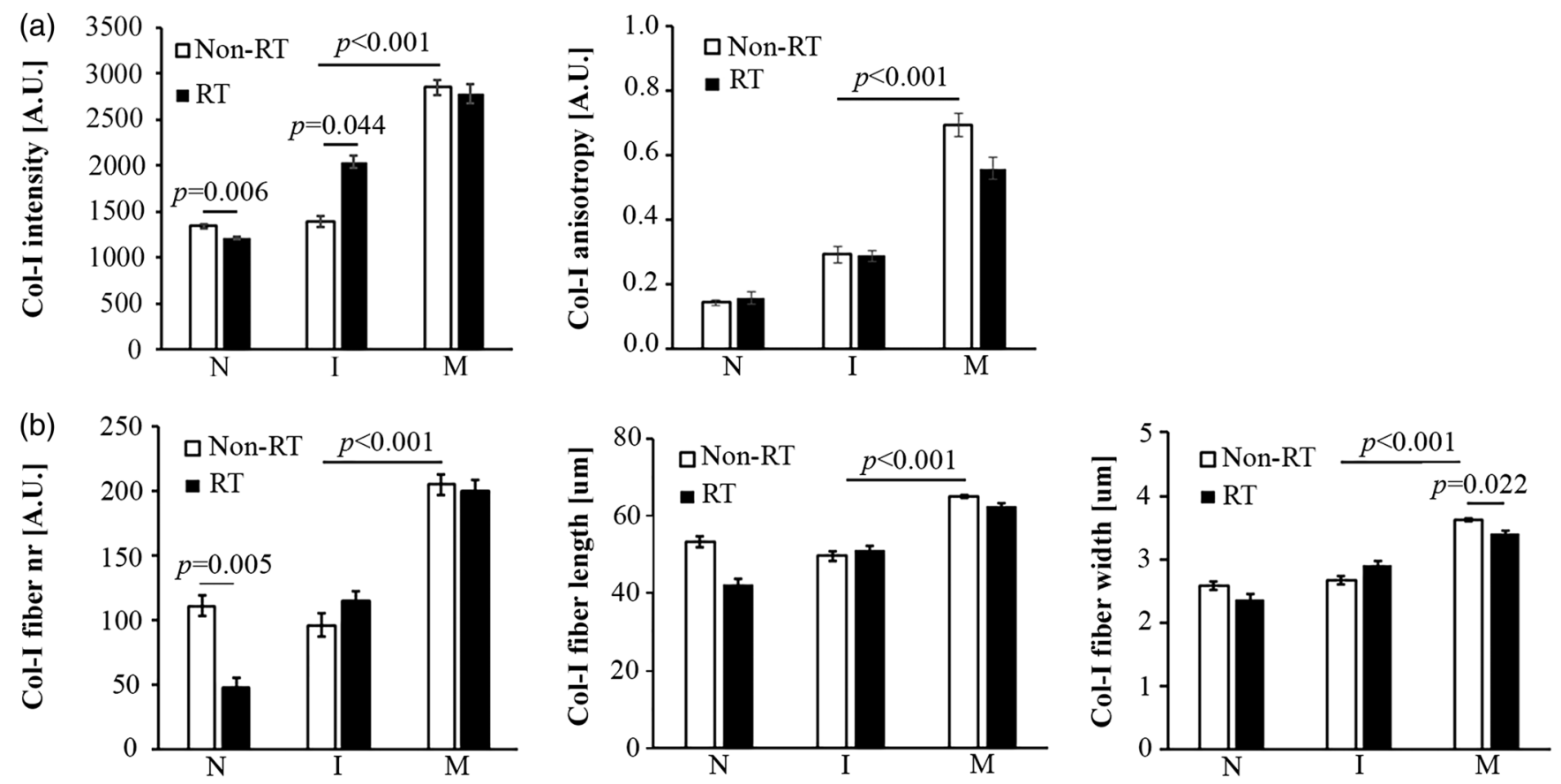

Fig. 2 Col-I characteristics in the different regions, namely adjacent normal mucosa (N), inner tumor (I), and invasive margin (M), of CDX2-stained surgical sections from non-RT and RT treated rectal cancer patients. (a) Col-I intensity and anisotropy. (b) Col-I fiber number, length, and width. Statistical significance of the differences between different tissue regions in non-RT condition, and between non-RT and RT conditions for each tissue region is represented with the $p$-value calculated using the two-tailed $t$-test. Error bar indicates the standard error of the mean. 
(a)
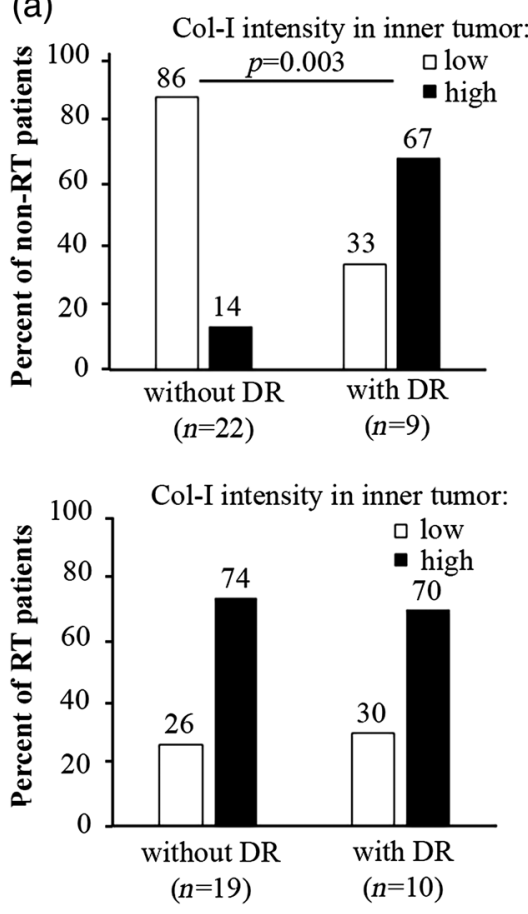

(b)
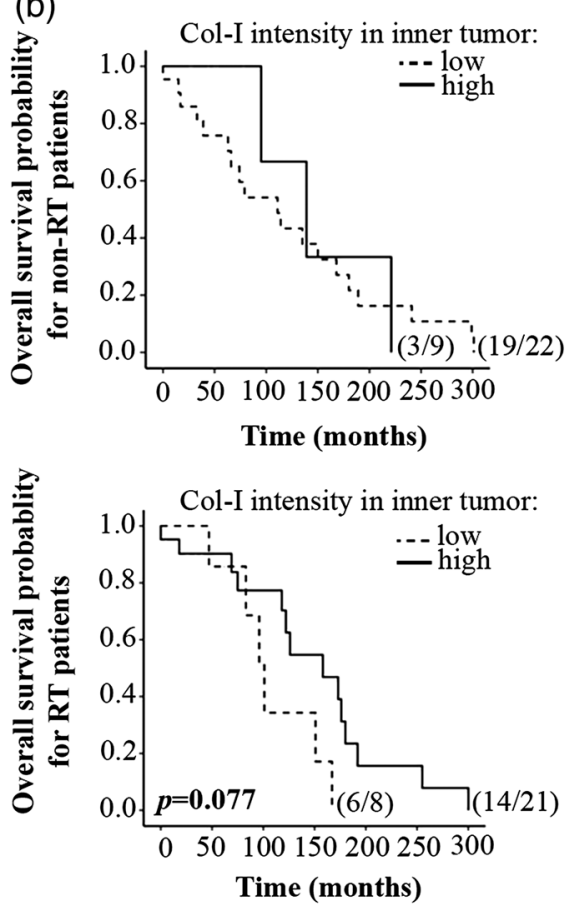

(c)
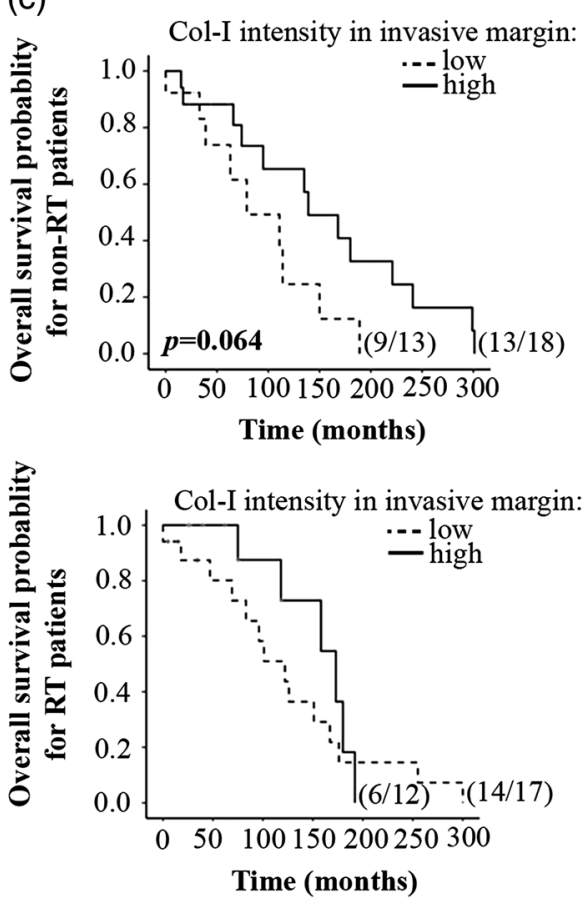

Fig. 3 Clinical relevance of Col-I intensity in surgical tissue of non-RT and RT rectal cancer patients. (a) Non-RT, but not RT, patients with high Col-I intensity in the inner tumor had an increased risk for developing distant recurrence (DR) $(p=0.003)$. (b) Non-RT, but not RT, patients with high Col-I intensity in the invasive margin had a higher overall survival rate $(p=0.064)$. (C) $\mathrm{RT}$, but not non-RT, patients with high Col-I intensity in the inner tumor had a higher overall survival rate $(p=0.077)$. The patient numbers are presented in parentheses.

positive relation between Col-I fiber width in tumor margin and the tumor differentiation grade in the non-RT group ( $p=0.010)$, and between Col-I fiber length in the tumor margin and the tumor stage in the RT group $(p=0.014)$.

Our findings for the non-RT patients correspond with the study reported by Nyström et al., ${ }^{23}$ where Col-I amount in stroma of CRC is positively related to the high grade of differentiation and the risk for metastatic lesions. We speculate that the contrasting clinical outcome for increased Col-I amount in the inner tumor region of non-RT and RT patients may be due to a different structure of the Col-I matrix. More highly ordered Col-I fibers may promote cancer cell invasion and thus metastasis. ${ }^{12,32}$

\section{Conclusions}

Our study demonstrates the strength of SHG microscopy for characterization of the Col-I matrix in surgical rectal tumor tissue. Several Col-I properties (including amount, anisotropy, fiber number, width, and length) could be defined by SHG. Under non-RT conditions, the Col-I amount and alignment were increased in the invasive margin versus the inner tumor. After RT, the Col-I amount was decreased in the normal mucosa while increased in the inner tumor. Further clinicopathological evaluation indicated that under non-RT condition, a higher Col-I amount in the inner tumor region is related to more distant recurrence, whereas after RT (followed by surgery), a higher Col-I amount in the inner tumor region predicts a longer patient survival. In the future, we will investigate the predictive value of various Col-I properties, that can be resolved by SHG, by comparing biopsy and surgical samples before and after RT, respectively.

\section{Disclosures}

The authors declare no conflict of interest.

\section{Acknowledgments}

The study was supported by grants from the Swedish Cancer Foundation, the Research Council of South East Sweden, and Liu Cancer; the Trygger Foundation; and, the Swedish Research Council and Knut och Alice Wallenberg Foundation. We thank Annika Enejder for giving us access to the nonlinear microscope and Juris Kiskis for his help with the CT-FIRE analysis of SHG images at the Department of Biology and Biological Engineering at Chalmers University of Technology.

\section{References}

1. J. Ferlay et al., "Cancer incidence and mortality worldwide: sources, methods and major patterns in GLOBOCAN 2012," Int. J. Cancer 136(5), E359-E386 (2015).

2. A. Jemal et al., "Global cancer statistics," CA: Cancer J. Clin. 61(2), 69-90 (2011).

3. L. Påhlman, "Indications for neoadjuvant long-term radiotherapy," in Recent Results in Cancer Research, M. W. Büchler et al., Eds., Vol. 165, pp. 212-220, Springer, Berlin, Heidelberg (2005).

4. Swedish Rectal Cancer Trial, "Improved survival with preoperative radiotherapy in resectable rectal cancer," N. Engl. J. Med. 336(14), 980-987 (1997).

5. E. Kapiteijn et al., "Preoperative radiotherapy combined with total mesorectal excision for resectable rectal cancer," N. Engl. J. Med. 345(9), 638-646 (2001).

6. S. Huerta, X. Gao, and D. Saha, "Mechanisms of resistance to ionizing radiation in rectal cancer," Expert Rev. Mol. Diagn. 9(5), 469-480 (2009). 
7. V. H. Koelzer and A. Lugli, "The tumor border configuration of colorectal cancer as a histomorphological prognostic indicator," Front. Oncol. 18(4), 29 (2014).

8. J. Gao et al., "Stromal staining for PINCH is an independent prognostic indicator in colorectal cancer," Neoplasia 6(6), 796-801 (2004).

9. H. Denys et al., "The extracellular matrix regulates cancer progression and therapy response: implications for prognosis and treatment," Curr. Pharm. Des. 15(12), 1373-1384 (2009).

10. P. Lu, V. M. Weaver, and Z. Werb, "The extracellular matrix: a dynamic niche in cancer progression," J. Cell Biol. 196(4), 395-406 (2012).

11. M. Fang et al., "Collagen as a double-edged sword in tumor progression," Tumor Biol. 35(4), 2871-2882 (2014).

12. P. P. Provenzano et al., "Collagen reorganization at the tumor-stromal interface facilitates local invasion," BMC Med. 4(1), 38 (2006).

13. M. W. Conklin et al., "Aligned collagen is a prognostic signature for survival in human breast carcinoma," Am. J. Pathol. 178(3), 12211232 (2011).

14. Y. Yarnold and M. C. Brotons, "Pathogenetic mechanisms in radiation fibrosis," Radiother. Oncol. 97(1), 149-161 (2010).

15. M. O'Neil and I. Damjanov, "Histopathology of colorectal cancer after neoadjuvant chemoradiation therapy," Open Pathol. J. 3, 91-98 (2009).

16. C. F. Von Essen, "Radiation enhancement of metastasis: a review," Clin. Exp. Metastasis 9(2), 77-104 (1991).

17. C. Moncharmont et al., "Radiation-enhanced cell migration/invasion process: a review," Crit. Rev. Oncol./Hematol. 92(2), 133-142 (2014).

18. S. Blockhuys et al., "X-radiation enhances the collagen type I strap formation and migration potentials of colon cancer cells," Oncotarget 7(44), 71390-71399 (2016).

19. R. Cicchi et al., "From molecular structure to tissue architecture: collagen organization probed by SHG microscopy," J. Biophotonics 6(2), 129-142 (2013).

20. V. E. Centonze and J. G. White, "Multiphoton excitation provides optical sections from deeper within scattering specimens than confocal imaging," Biophys. J. 75(4), 2015-2024 (1998).

21. J. W. Birk et al., "Second harmonic generation imaging distinguishes both high-grade dysplasia and cancer from normal colonic mucosa," Dig. Dis. Sci. 59(7), 1529-1534 (2014).

22. J. Adur et al., "Colon adenocarcinoma diagnosis in human samples by multicontrast nonlinear optical microscopy of hematoxylin and eosin stained histological sections," J. Cancer Ther. 5(13), 1259-1269 (2014).

23. H. Nyström et al., "Liver-metastatic potential of colorectal cancer is related to the stromal composition of the tumour," Anticancer Res. 32(12), 5183-5191 (2012).

24. Swedish Rectal Cancer Trial, "Initial report from a Swedish multicenter study examining the role of preoperative irradiation in the treatment of patients with resectable rectal carcinoma," Br. J. Surg. 80(10), 13331336 (1993).

25. A. Boudaoud et al., "FibrilTool, an ImageJ plug-in to quantify fibrillar structures in raw microscopy images," Nat. Protoc. 9(2), 457-463 (2014).

26. J. S. Bredfeldt et al., "Computational segmentation of collagen fibers from second-harmonic generation images of breast cancer," J. Biomed. Opt. 19(1), 016007 (2014).

27. A. C. Chen et al., "Second harmonic generation and multiphoton microscopic detection of collagen without the need for species specific antibodies," Burns 37(6), 1001-1009 (2011).
28. R. S. Saad et al., "CDX2 as a marker for intestinal differentiation: its utility and limitations," World J. Gastrointest. Surg. 3(11), 159-166 (2011).

29. R. Kalluri and M. Zeisberg, "Fibroblasts in cancer," Nat. Rev. Cancer 6 , 392-401 (2006)

30. A. Kumar et al., "Increased type-IV collagenase (MMP-2 and MMP-9) activity following preoperative radiotherapy in rectal cancer," $\mathrm{Br}$. $J$. Cancer 82(4), 960-965 (2000).

31. X. Chen et al., "Second harmonic generation microscopy for quantitative analysis of collagen fibrillar structure," Nat. Protoc. 7(4), 654-669 (2012).

32. J. B. Wyckoff et al., "Direct visualization of macrophage-assisted tumor cell intravasation in mammary tumors," Cancer Res. 67(6), 2649-2656 (2007).

Stéphanie Blockhuys has been a postdoctoral researcher at Chalmers since 2015. She obtained her PhD in medical sciences in 2013 at Ghent University, Belgium. She moved to Sweden in 2013 for a first postdoc at Linköping University, followed by a second postdoc at Chalmers (her current position). She is an all-around cancer researcher with bench-to-bedside experience in breast and colorectal cancer, and she strives for a better fundamental understanding of cancer by application of novel technologies.

Nisha Rani Agarwal is a postdoctoral researcher at Biointerfaces Institute at McMaster University, Canada, working with colorimetric biosensing of paper-based POC devices. She received her $\mathrm{PhD}$ in materials engineering in 2013 at Politecnico di Milano for controlled synthesis of noble metal SERS substrates by pulsed laser deposition as biosensors. She pursued a postdoc at Chalmers University of Technology, specializing in microscopy techniques as CARS, SHG, AFM, SNOM, and TERS for biological and plasmonic applications.

Camilla Hildesjö obtained her title as medical biologist in 2008 (Med. Licentiate - Keratinocytes in tissue engineering, in-vitro and in-vivo studies). She is currently working for the Department of Clinical and Experimental Sciences, Linköping University, and for the Department of Clinical Pathology, Diagnostikcentrum Region Östergötlands. She specializes in colorectal cancers, with focus on biomarkers for the prediction of therapy outcome and overall survival of patients with colorectal cancer.

Ingvar Jarlsfelt is medical director of clinical physiology, laboratory medicine and $x$-ray at Ryhov hospital in Jönköping.

Pernilla Wittung-Stafshede is a professor and division chair at Chalmers. She obtained her PhD in 1996. After a postdoc at California Institute of Technology, she became assistant professor at Tulane University, New Orleans, in 1999. She received tenure in 2002. In 2004, she moved to Rice University, Houston, as an associate professor with tenure. In 2008, she returned to Sweden and became professor at Umeå University. After seven years, she moved to her current position.

Xiao-Feng Sun has been a professor at Linköping University since 2005. She obtained her medical degree in 1982 and her $\mathrm{PhD}$ in 1993 and became project leader of colorectal cancer research at Linköping University in 1996 (until present). 